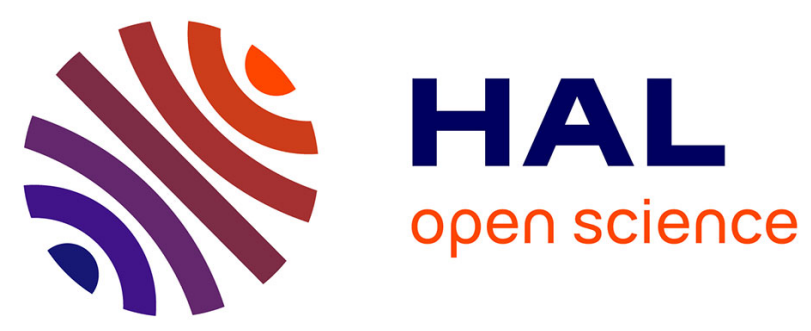

\title{
Inducing an order on cellular automata by a grouping operation.
}

\author{
Jacques Mazoyer, Ivan Rapaport
}

\section{To cite this version:}

Jacques Mazoyer, Ivan Rapaport. Inducing an order on cellular automata by a grouping operation.. [Research Report] LIP RR-1997-33, Laboratoire de l'informatique du parallélisme. 1997, 2+16p. hal02101944

\section{HAL Id: hal-02101944 \\ https://hal-lara.archives-ouvertes.fr/hal-02101944}

Submitted on 17 Apr 2019

HAL is a multi-disciplinary open access archive for the deposit and dissemination of scientific research documents, whether they are published or not. The documents may come from teaching and research institutions in France or abroad, or from public or private research centers.
L'archive ouverte pluridisciplinaire HAL, est destinée au dépôt et à la diffusion de documents scientifiques de niveau recherche, publiés ou non, émanant des établissements d'enseignement et de recherche français ou étrangers, des laboratoires publics ou privés. 


\section{Laboratoire de l'Informatique du Parallélisme}

Ecole Normale Supérieure de Lyon

Unité de recherche associée au CNRS n ${ }^{\circ} 1398$

\section{Inducing an Order on Cellular Automata by a Grouping Operation}

Jacques Mazoyer

Ivan Rapaport

Research Report $\mathrm{N}^{\mathrm{O}} 97-33$

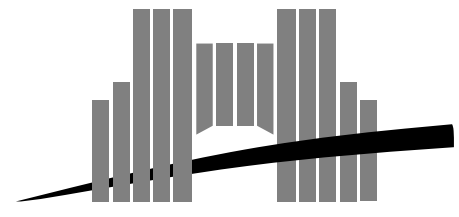

Ecole Normale Supérieure de Lyon

46 Allée d'Italie, 69364 Lyon Cedex 07, France

Téléphone : (+33) (0)4.72.72.80.00 Télécopieur : (+33) (0)4.72.72.80.80

Adresse électronique : lip@lip.ens-lyon.fr 


\title{
Inducing an Order on Cellular Automata by a Grouping Operation
}

\author{
Jacques Mazoyer \\ Ivan Rapaport
}

September 1997

\begin{abstract}
A grouped instance of a cellular automaton (CA) is another one obtained by grouping several states into blocks and by letting interact neighbor blocks. Based on this operation (and on the subautomaton notion), a preorder $\leq$ on the set of one dimensional $\mathrm{CA}$ is introduced. It is shown that $(\mathrm{CA}, \leq)$ admits a global minimum and that on the bottom of $(\mathrm{CA}, \leq)$ very natural equivalence classes are located. These classes remind us the first two well-known Wolfram ones because they capture global (or dynamical) properties as nilpotency or periodicity. Non trivial properties as the undecidability of $\leq$ and the existence of bounded infinite chains are also proved. Finally, it is shown that $(\mathrm{CA}, \leq)$ admits no maximum. This result allows us to conclude that, in a "grouping sense", there is no universal CA.
\end{abstract}

Keywords: cellular automata, grouping, order, dynamical classification, intrinsic real time universality.

\section{Résumé}

Une instance groupeé d'un automate cellulaire (AC) est un autre obtenu par groupage de plusieurs états en blocs et par l'interaction "naturelle" de ces blocs. Basé sur cette opération (et sur la notion de sous-automate), un préordre $\leq$ sur l'ensamble des $\mathrm{AC}$ à une dimension est introduit. Il est montré que $(\mathrm{AC}, \leq)$ admet un minimum global et que des classes d'equivalence très naturelles se trouvent en bas de $(\mathrm{AC}, \leq)$. On retrouve dans ces classes les deux premières bien connues de Wolfram qui capturent des propietés globales (ou dynamiques) comme la nilpotence et la périodicité. Des propietés non triviales comme l'indécidabilité de $\leq$ et l'existence de chaines infinies bornées sont aussi prouvées. Finalement il est montré que $(\mathrm{AC}, \leq)$ n'admet pas de maximum. Cet resultat nous permet de conclure qu'il n'existe pas de AC universel d'un point de vue "groupage".

Mots-clés: automates cellulaires, groupage, ordre, classification dynamique, universalité intrinsèque en temps réel. 


\title{
Inducing an Order on Cellular Automata by a Grouping Operation *
}

\author{
Jacques Mazoyer Iván Rapaport \\ LIP-École Normale Supérieure de Lyon \\ 46 Allée d'Italie, 69364 Lyon Cedex 07 FRANCE \\ e-mail: \{mazoyer,irapapor\}@lip.ens-lyon.fr
}

\begin{abstract}
A grouped instance of a cellular automaton (CA) is another one obtained by grouping several states into blocks and by letting interact neighbor blocks. Based on this operation (and on the subautomaton notion), a preorder $\leq$ on the set of one dimensional $\mathrm{CA}$ is introduced. It is shown that $(\mathrm{CA}, \leq)$ admits a global minimum and that on the bottom of $(\mathrm{CA}, \leq)$ very natural equivalence classes are located. These classes remind us the first two well-known Wolfram ones because they capture global (or dynamical) properties as nilpotency or periodicity. Non trivial properties as the undecidability of $\leq$ and the existence of bounded infinite chains are also proved. Finally, it is shown that $(\mathrm{CA}, \leq)$ admits no maximum. This result allows us to conclude that, in a "grouping sense", there is no universal CA.
\end{abstract}

Key words: cellular automata, grouping, order, dynamical classification, intrinsic real time universality.

\section{Introduction}

A one dimensional cellular automaton with unitary radius, or simply a CA, is an infinite array of finite state machines called cells and indexed by $\mathbb{Z}$. These identical cells evolve synchronously at discrete time steps following a local rule by which the state of a cell is determined as a function of its own state together with the states of its two neighbors. These devices are capable to simulate any Turing machine ([7]) and, despite their simplicity, they may exhibit very complex behavior. The goal of this paper is to introduce an order on the set of $\mathrm{CA}$ by considering them as algebraic objects.

A first approach is to say that a $\mathrm{CA} A$ is a subautomaton of a $\mathrm{CA} B$ if the transition table of $A$ is contained (after a suitable relabeling of the states) on the transition table of $B$. This notion takes into account only the finite-state-machine nature of the CA cells but not their spatial dimension.

On the other hand, the evolution of a CA from a particular initial configuration is usually represented by a space-time diagram in $\mathbb{Z}^{2}$. When observing it, the human eye typically

${ }^{*}$ This work was partially supported by Chilean-French Cooperation Programs (specially ECOS-97). 
changes the scale in order to remove irrelevant microscopic details and to discover a macroscopic behavior. We call to the CA that generate this scaled space-time diagrams grouped instances (or powers) of the original one. They are obtained by grouping several states into blocks and by considering as transitions the interactions of neighbor blocks. In section 2 this definition is formally stated.

In section 3 it is shown that previous grouping operation (together with the subautomaton notion) effectively allows us to introduce a preorder $\leq$ on the set of $\mathrm{CA}$. In fact, it suffices to note $A \leq B$ when a grouped instance of $A$ is a subautomaton of a grouped instance of $B$. This preorder induces a canonical equivalence relation. More precisely, we say that two $\mathrm{CA}$ $A$ and $B$ are equivalent if $A \leq B$ and $B \leq A$. Notice that, as we expected it, at least all the grouped instances of a $\mathrm{CA}$ are equivalent.

In section 4 we show that $(\mathrm{CA}, \leq)$ admits a global minimum and that on the bottom of $(\mathrm{CA}, \leq)$ very natural equivalence classes are located. These classes remind us the first two well-known Wolfram ones ([8]) because they capture global (or dynamical) properties as nilpotency or periodicity. As a corollary, the undecidability of $\leq$ is stated.

In section 5 a non trivial property concerning $(\mathrm{CA}, \leq)$ is proved: the existence of two incomparable infinite chains with a common upper bound.

Finally, in section 6 a natural question is answered. In fact, it is shown that $(\mathrm{CA}, \leq)$ has no maximum. In other words, for any $\mathrm{CA}$, the subsystems of all its grouped instances will never cover all the CA classes. This result allows us to conclude that, in a "grouping sense", there is no universal CA.

\section{Definitions}

Formally, a CA is a couple $(Q, \delta)$ where $Q$ is a finite set of states and $\delta: Q^{3} \rightarrow Q$ is a transition function. A configuration of a $\mathrm{CA}(Q, \delta)$ is a bi-infinite sequence $\mathcal{C} \in Q^{\mathbb{Z}}$, and its global transition function $G_{\delta}: Q^{\mathbb{Z}} \rightarrow Q^{\mathbb{Z}}$ is such that $\left(G_{\delta}(\mathcal{C})\right)_{i}=\delta\left(\mathcal{C}_{i-1}, \mathcal{C}_{i}, \mathcal{C}_{i+1}\right)$.

Given a particular state $s \in Q$, we denote its corresponding homogeneous configuration as $\bar{s}=(\cdots, s, s, s, \cdots) \in Q^{\mathbb{Z}}$. We denote $\mathbb{N}^{*}=\mathbb{N}-\{0\}$ and, to each $n \in N^{*}$, we associate the set of states $S_{n}=\{0, \cdots, n-1\}$.

We say that $\left(Q_{1}, \delta_{1}\right)$ is a subautomaton of $\left(Q_{2}, \delta_{2}\right)$, and we note $\left(Q_{1}, \delta_{1}\right) \subseteq\left(Q_{2}, \delta_{2}\right)$, if there exists an injection $\varphi: Q_{1} \rightarrow Q_{2}$ such that for all $x, y, z \in Q_{1}: \varphi\left(\delta_{1}(x, y, z)\right)=$ $\delta_{2}(\varphi(x), \varphi(y), \varphi(z))$. When the function $\varphi$ is a bijection we say that $\left(Q_{1}, \delta_{1}\right)$ and $\left(Q_{2}, \delta_{2}\right)$ are isomorph and we note $\left(Q_{1}, \delta_{1}\right) \cong\left(Q_{2}, \delta_{2}\right)$.

For any $\mathrm{CA}(Q, \delta)$ the evolution of a finite block of states looks like a light-cone (see figure 1). This basic fact inspires the notion of the $n$-block evolution function $\delta^{n}: Q^{2 n+1} \rightarrow Q$, which is recursively defined as follows:

$$
\begin{aligned}
\delta^{1}\left(w_{-1}, w_{0}, w_{1}\right) & =\delta\left(w_{-1}, w_{0}, w_{1}\right) \\
\delta^{n}\left(w_{-n} \cdots, w_{0}, \cdots, w_{n}\right) & =\delta^{n-1}\left(\delta\left(w_{-n}, w_{-n+1}, w_{-n+2}\right) \cdots \delta\left(w_{n-2}, w_{n-1}, w_{n}\right)\right)
\end{aligned}
$$




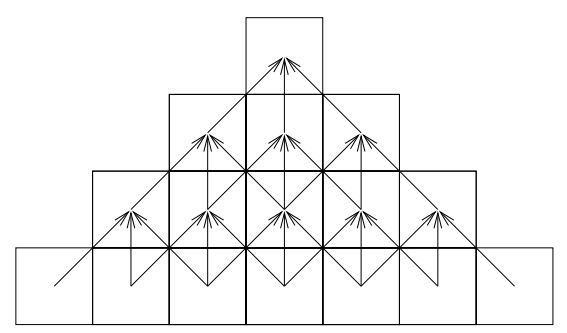

Fig. 1 Dependences diagram representing a block of states evolution as a light-cone.

By grouping several states into blocks and by letting interact triplets of blocks as schematically appears in figure 2, we generate CA with (exponentially) more states.

Formally, the $n$-grouped instance of a $\mathrm{CA}(Q, \delta)$ is the $\mathrm{CA}(Q, \delta)^{n}=\left(Q^{n}, \delta_{\mathrm{g}}^{n}\right)$, where $\vec{q} \in Q^{n}$ is denoted $\left(q_{1}, \cdots, q_{n}\right)$ and for all $\vec{x}, \vec{y}, \vec{z} \in Q^{n}$ :

$$
\left(\delta_{\mathrm{g}}^{n}(\vec{x}, \vec{y}, \vec{z})\right)_{i}=\delta^{n}\left(x_{i}, \cdots, x_{n}, y_{1}, \cdots, y_{i}, \cdots, y_{n}, z_{1}, \cdots, z_{i}\right)
$$

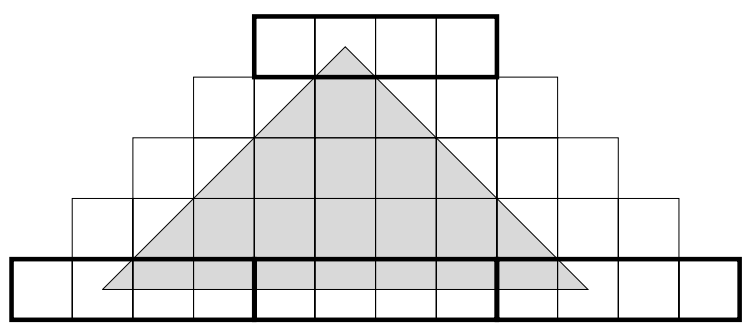

Fig. 2 Blocks interaction.

Finally we introduce a binary relation on the set of $\mathrm{CA}$. It is denoted as $\leq$ and it associates two $\mathrm{CA}$ when a grouped instance of the first is a subautomaton of a grouped instance of the second. More precisely, for two CA $\left(Q_{1}, \delta_{1}\right)$ and $\left(Q_{2}, \delta_{2}\right)$,

$$
\left(Q_{1}, \delta_{1}\right) \leq\left(Q_{2}, \delta_{2}\right) \Longleftrightarrow \exists n, m \in \mathbb{N}^{*}:\left(Q_{1}, \delta_{1}\right)^{n} \subseteq\left(Q_{2}, \delta_{2}\right)^{m}
$$

\section{An Order on CA}

Here we show that the relation $\leq$ is a preorder on $\mathrm{CA}$. This preorder induces a canonical equivalence relation and it satisfies two basic properties proved at the end of the section. The first one says that all the grouped instances of a given CA are $\leq$-equivalent, and the second one that by "superposing" two CA we obtain a common maximum.

Lemma 1 Let $(Q, \delta)$ be a CA. For all $i, n \in \mathbb{N}^{*}$ such that $i<n$ it holds:

$$
\delta^{n}\left(w_{-n}, \cdots, w_{n}\right)=\delta^{n-i}\left(\delta^{i}\left(w_{-n}, \cdots, w_{-n+2 i}\right), \delta^{i}\left(w_{-n+1}, \cdots, w_{-n+2 i+1}\right), \cdots, \delta^{i}\left(w_{n-2 i}, \cdots, w_{n}\right)\right)
$$

Proof By induction.

Lemma 2 Let $\left(Q_{1}, \delta_{1}\right) \subseteq\left(Q_{2}, \delta_{2}\right)$ be two CA. For all $n \in I N^{*}$ it holds:

$$
\left(Q_{1}, \delta_{1}\right)^{n} \subseteq\left(Q_{2}, \delta_{2}\right)^{n}
$$


Proof Let $\varphi: Q_{1} \rightarrow Q_{2}$ be a suitable injection. First we have to prove by induction on $n$ that $\varphi\left(\delta_{1}^{n}\left(w_{-n}, \cdots, w_{n}\right)\right)=\delta_{2}^{n}\left(\varphi\left(w_{-n}\right), \cdots, \varphi\left(w_{n}\right)\right)$. It is direct for $n=1$. Assuming it true for $n$, and denoting $A_{n+1}=\varphi\left(\delta_{1}^{n+1}\left(w_{-n-1}, \cdots, w_{n+1}\right)\right)$, it follows:

$$
\begin{aligned}
A_{n+1} & =\varphi\left(\delta_{1}^{n}\left(\delta_{1}\left(w_{-n-1}, w_{-n}, w_{-n+1}\right), \cdots, \delta_{1}\left(w_{n-1}, w_{n}, w_{n+1}\right)\right)\right) \\
& =\delta_{2}^{n}\left(\varphi\left(\delta_{1}\left(w_{-n-1}, w_{-n}, w_{-n+1}\right)\right), \cdots, \varphi\left(\delta_{1}\left(w_{n-1}, w_{n}, w_{n+1}\right)\right)\right) \\
& =\delta_{2}^{n}\left(\delta_{2}\left(\varphi\left(w_{-n-1}\right), \varphi\left(w_{-n}\right), \varphi\left(w_{-n+1}\right)\right), \cdots, \delta_{2}\left(\varphi\left(w_{n-1}\right), \varphi\left(w_{n}\right), \varphi\left(w_{n+1}\right)\right)\right) \\
& =\delta_{2}^{n+1}\left(\varphi\left(w_{n-1}\right), \cdots, \varphi\left(w_{n+1}\right)\right)
\end{aligned}
$$

Let us consider now the injection $\vec{\varphi}:\left(Q_{1}\right)^{n} \rightarrow\left(Q_{2}\right)^{n}$ such that $\vec{\varphi}(\vec{x})=\left(\varphi\left(x_{1}\right), \cdots, \varphi\left(x_{n}\right)\right)$. It follows that for all $\vec{x}, \vec{y}, \vec{z} \in\left(Q_{1}\right)^{n}$ :

$$
\begin{aligned}
\left(\vec{\varphi}\left(\left(\delta_{1}\right)_{\mathrm{g}}^{n}(\vec{x}, \vec{y}, \vec{z})\right)_{i}\right. & =\varphi\left(\delta_{1}^{n}\left(x_{i}, \cdots, y_{i}, \cdots, z_{i}\right)\right) \\
& =\delta_{2}^{n}\left(\varphi\left(x_{i}\right), \cdots, \varphi\left(y_{i}\right), \cdots, \varphi\left(z_{i}\right)\right) \\
& =\left(\left(\delta_{2}\right)_{\mathrm{g}}^{n}(\vec{\varphi}(\vec{x}), \vec{\varphi}(\vec{y}), \vec{\varphi}(\vec{z}))_{i}\right.
\end{aligned}
$$

Lemma 3 Let $(Q, \delta)$ be a CA. For all $n, m \in N^{*}$ it holds:

$\left((Q, \delta)^{n}\right)^{m} \cong(Q, \delta)^{n m}$

Proof Denoting $\vec{a} \in\left(Q^{n}\right)^{m}$ as $\left(\vec{a}_{1}, \cdots, \vec{a}_{m}\right)$ with $\vec{a}_{i}=\left(a_{i 1}, \cdots, a_{i n}\right) \in Q^{n}$ and $\vec{b} \in Q^{n m}$ as $\left(b_{11}, \cdots, b_{1 n}, \cdots \cdots, b_{m 1}, \cdots, b_{m n}\right)$ with $b_{i j} \in Q$, and defining the bijection $\varphi:\left(Q^{n}\right)^{m} \rightarrow Q^{n m}$ such that $(\varphi(\vec{a}))_{i j}=\left(\vec{a}_{i}\right)_{j}$, in order to prove the lemma it suffices to show that next identity holds for all $i \leq m, j \leq n$ and for all $\vec{x}, \vec{y}, \vec{z} \in\left(Q^{n}\right)^{m}$ :

$\left[\left(\delta_{\mathrm{g}}^{n}\right)^{m}\left(\vec{x}_{i}, \cdots, \vec{x}_{m}, \vec{y}_{1}, \cdots, \vec{y}_{m}, \vec{z}_{1}, \cdots, \vec{z}_{i}\right)\right]_{j}=\delta^{n m}\left(\left(\vec{x}_{i}\right)_{j}, \cdots,\left(\vec{x}_{m}\right)_{n},\left(\vec{y}_{1}\right)_{1}, \cdots,\left(\vec{y}_{m}\right)_{n},\left(\vec{z}_{1}\right)_{1}, \cdots,\left(\vec{z}_{i}\right)_{j}\right)$

because

$$
\left[\varphi\left(\left(\delta_{\mathrm{g}}^{n}\right)_{\mathrm{g}}^{m}(\vec{x}, \vec{y}, \vec{z})\right)\right]_{i j}=\left[\left(\left(\delta_{\mathrm{g}}^{n}\right)_{\mathrm{g}}^{m}(\vec{x}, \vec{y}, \vec{z})\right)_{i}\right]_{j}=\left[\left(\delta_{\mathrm{g}}^{n}\right)^{m}\left(\vec{x}_{i}, \cdots, \vec{x}_{m}, \vec{y}_{1}, \cdots, \vec{y}_{m}, \vec{z}_{1}, \cdots, \vec{z}_{i}\right)\right]_{j}
$$
and $\left[\delta_{\mathrm{g}}^{n m}(\varphi(\vec{x}), \varphi(\vec{y}), \varphi(\vec{z}))\right]_{i j}=\delta^{n m}\left((\varphi(\vec{x}))_{i j}, \cdots,(\varphi(\vec{z}))_{i j}\right)=\delta^{n m}\left(\left(\vec{x}_{i}\right)_{j}, \cdots,\left(\vec{z}_{i}\right)_{j}\right)$

We prove finally the identity by induction on $m$. For $m=1$ it holds directly. Assuming it true for $m$, it follows:

$$
\begin{aligned}
{\left[\left(\delta_{\mathrm{g}}^{n}\right)^{m+1}\left(\vec{x}_{i}, \cdots, \vec{z}_{i}\right)\right]_{j} } & =\left[\left(\delta_{\mathrm{g}}^{n}\right)^{m}\left(\delta_{\mathrm{g}}^{n}\left(\vec{x}_{i}, \vec{x}_{i+1}, \vec{x}_{i+2}\right), \cdots, \delta_{\mathrm{g}}^{n}\left(\vec{z}_{i-2}, \vec{z}_{i-1}, \vec{z}_{i}\right)\right)\right]_{j} \\
& =\delta^{n m}\left(\left(\delta_{\mathrm{g}}^{n}\left(\vec{x}_{i}, \vec{x}_{i+1}, \vec{x}_{i+2}\right)\right)_{j}, \cdots,\left(\delta_{\mathrm{g}}^{n}\left(\vec{z}_{i-2}, \vec{z}_{i-1}, \vec{z}_{i}\right)\right)_{j}\right) \\
& =\delta^{n m}\left(\delta^{n}\left(\left(\vec{x}_{i}\right)_{j}, \cdots,\left(\vec{x}_{i+2}\right)_{j}\right), \cdots, \delta^{n}\left(\left(\vec{z}_{i-2}\right)_{j}, \cdots,\left(\vec{z}_{i}\right)_{j}\right)\right) \\
& =\delta^{n(m+1)}\left(\left(\vec{x}_{i}\right)_{j}, \cdots,\left(\vec{z}_{i}\right)_{j}\right)
\end{aligned}
$$

Proposition 1 The relation $\leq$ is a preorder on $\mathrm{CA}$.

Proof The reflexivity holds directly. For the transitivity, let us consider $\left(Q_{1}, \delta_{1}\right) \leq\left(Q_{2}, \delta_{2}\right)$ and $\left(Q_{2}, \delta_{2}\right) \leq\left(Q_{3}, \delta_{3}\right)$. By definition, there exist $n_{1}, m_{1}, n_{2}, m_{2} \in \mathbb{N}^{*}$ such that $\left(Q_{1}, \delta_{1}\right)^{n_{1}} \subseteq$ $\left(Q_{2}, \delta_{2}\right)^{m_{1}}$ and $\left(Q_{2}, \delta_{2}\right)^{n_{2}} \subseteq\left(Q_{3}, \delta_{3}\right)^{m_{2}}$. By applying lemma 2 and lemma 3 , together with the transitivity of $\subseteq$, we conclude that $\left(Q_{1}, \delta_{1}\right)^{n_{1} n_{2}} \subseteq\left(Q_{3}, \delta_{3}\right)^{m_{1} m_{2}}$. 
Remark 1 The preorder $\leq$ defined on CA induces:

i. An equivalence relation $\sim$ on $\mathrm{CA}$, where:

$$
\left[\left(Q_{1}, \delta_{1}\right) \sim\left(Q_{2}, \delta_{2}\right)\right] \Leftrightarrow\left[\left(Q_{1}, \delta_{1}\right) \leq\left(Q_{2}, \delta_{2}\right) \text { and }\left(Q_{2}, \delta_{2}\right) \leq\left(Q_{1}, \delta_{1}\right)\right]
$$

ii. A strict preorder $<$ on $\mathrm{CA}$, where:

$$
\left[\left(Q_{1}, \delta_{1}\right)<\left(Q_{2}, \delta_{2}\right)\right] \Leftrightarrow\left[\left(Q_{1}, \delta_{1}\right) \leq\left(Q_{2}, \delta_{2}\right) \text { and }\left(Q_{1}, \delta_{1}\right) \not\left(Q_{2}, \delta_{2}\right)\right]
$$

iii. The canonical order on $(\mathrm{CA} / \sim)$ compatible with $\leq$.

Proposition 2 For any $\mathrm{CA}(Q, \delta)$, all its grouped instances are equivalent. In other words, for all $i, j \in \mathbb{I N}^{*}:(Q, \delta)^{i} \sim(Q, \delta)^{j}$.

Proof It suffices to notice that by lemma $3,\left((Q, \delta)^{j}\right)^{i} \sim(Q, \delta)^{i j} \sim\left((Q, \delta)^{i}\right)^{j}$.

In order to obtain a local maximum for a finite family of $\mathrm{CA}$, let us consider the following "superposition" operation:

Definition 1 Let $\{B\}$ be such that $\{B\} \nsubseteq Q$ for every $\mathrm{CA}(Q, \delta)$. Let $\left(Q_{1}, \delta_{1}\right),\left(Q_{2}, \delta_{2}\right)$ be two $C A$. Then $(Q, \delta)=\left(Q_{1}, \delta_{1}\right) \otimes\left(Q_{2}, \delta_{2}\right)$ if $Q=\left(Q_{1} \cup\{B\}\right) \times\left(Q_{2} \cup\{B\}\right)$ and for all $\vec{x}=\left(x_{1}, x_{2}\right), \vec{y}=\left(y_{1}, y_{2}\right), \vec{z}=\left(z_{1}, z_{2}\right) \in Q$ :

$$
\delta(\vec{x}, \vec{y}, \vec{z})= \begin{cases}\left(\delta_{1}\left(x_{1}, y_{1}, z_{1}\right), B\right) & \text { if }(\vec{x}, \vec{y}, \vec{z}) \in\left(Q_{1} \times\{B\}\right)^{3} \\ \left(B, \delta_{2}\left(x_{2}, y_{2}, z_{2}\right)\right) & \text { if }(\vec{x}, \vec{y}, \vec{z}) \in\left(\{B\} \times Q_{2}\right)^{3} \\ (B, B) & \text { otherwise }\end{cases}
$$

Proposition 3 For every two $\mathrm{CA}\left(Q_{1}, \delta_{1}\right)$ and $\left(Q_{2}, \delta_{2}\right)$ the $\mathrm{CA}(Q, \delta)=\left(Q_{1}, \delta_{1}\right) \otimes\left(Q_{2}, \delta_{2}\right)$ satisfies $\left(Q_{1}, \delta_{1}\right) \leq(Q, \delta)$ and $\left(Q_{2}, \delta_{2}\right) \leq(Q, \delta)$.

Proof It suffices to consider the injections $\varphi_{1}: Q_{1} \rightarrow Q$ with $\varphi\left(x_{1}\right)=\left(x_{1}, B\right)$ and $\varphi_{2}:$ $Q_{2} \rightarrow Q$ with $\varphi\left(x_{2}\right)=\left(B, x_{2}\right)$.

\section{On the Bottom of $(\mathrm{CA}, \leq)$}

In this section we study the equivalence classes represented by the simplest CA. We show that for trivial transition functions (constant, identity, shift) very natural classes which capture global (or dynamical) properties as periodicity or nilpotency are generated. The undecidability of $\leq$ is concluded. In order to localize previous classes on the bottom of $(\mathrm{CA}, \leq)$ we must start by showing that $(\mathrm{CA}, \leq)$ effectively has a bottom. In fact, next proposition says that there exists a global minimum consisting on all the isomorph CA having only one state:

Proposition 4 The canonical order on $(\mathrm{CA} / \sim)$ compatible with $\leq$ admits a global minimum which corresponds to the class of $\mathrm{CA}$ having a single state.

Proof Let $\left(\{s\}, \delta_{s}\right)$ be such that $\delta_{s}(s, s, s)=s$. Let $(Q, \delta)$ be a CA. By the finiteness of $Q$ there exist $\tilde{q} \in Q$ and $P \in \mathbb{N}^{*}$ with $1 \leq P \leq|Q|$ such that $\delta^{P}(\tilde{q}, \cdots, \tilde{q})=\tilde{q}$, and therefore $\left(\{s\}, \delta_{s}\right) \subseteq(Q, \delta)^{P}$. Finally notice that if $|Q|>1$ then $\left(\{s\}, \delta_{s}\right)<(Q, \delta)$ because any grouped instance of a singleton $\mathrm{CA}$ is also a singleton $\mathrm{CA}$. 


\subsection{Nilpotency}

For a $\mathrm{CA}(Q, \delta)$ its limit set $\Omega(Q, \delta)$ is defined as the set of all the configurations that can occur after arbitrarily many computation steps. More precisely, if we define $\Omega^{0}=Q^{\mathbb{Z}}$ and $\Omega^{i}=G_{\delta}\left(\Omega^{i-1}\right)$ for $i \geq 1$, then $\Omega(Q, \delta)=\bigcap_{i=1}^{\infty} \Omega^{i}$. We say that a CA belongs to the class NIL, and we call it nilpotent, if its limit set is a singleton. In other words,

$$
N I L=\{(Q, \delta):(|Q|>1) \wedge(|\Omega(Q, \delta)|=1)\}
$$

Obviously, when the limit set is a singleton it corresponds to an homogeneous configuration. In [2] it is proved that when nilpotency holds then this configuration is reached from any other one in a finite and fixed number of steps. More precisely,

$$
N I L=\left\{(Q, \delta):(|Q|>1) \wedge\left(\exists s_{0} \in Q, n \in N^{*}\right)\left(\forall \mathcal{C} \in Q^{\mathbb{Z}}\right)\left(G_{\delta}^{n}(\mathcal{C})=\bar{s}_{0}\right)\right\}
$$

We introduce now the simplest nilpotent $\mathrm{CA}$ : those reaching the homogeneous configuration in one step. Let therefore $n>1$. The $\mathrm{CA}\left(S_{n}, 0_{n}\right)$ is such that for all $x, y, z \in S_{n}$ : $0_{n}(x, y, z)=0$.

Lemma 4 For all $n>1$ it holds: $\left(S_{2}, 0_{2}\right) \sim\left(S_{n}, 0_{n}\right)$.

Proof First notice that if $p \leq q$ then $0_{p}=\left.0_{q}\right|_{S_{p}}$, and therefore $\left(S_{p}, 0_{p}\right) \subseteq\left(S_{q}, 0_{q}\right)$. Let $n>1$ and let $\tilde{n} \in I^{*}$ be such that $n \leq 2^{\tilde{n}}$. It follows that $\left(S_{n}, 0_{n}\right) \subseteq\left(S_{2}, 0_{2}\right)^{\tilde{n}}$ because $\left(S_{2}, 0_{2}\right)^{\tilde{n}} \cong\left(S_{2^{\tilde{n}}}, 0_{2^{\tilde{n}}}\right)$.

Lemma 5 If $(Q, \delta) \leq\left(S_{2}, 0_{2}\right)$ then $(Q, \delta) \in N I L$ or $|Q|=1$.

Proof If $(Q, \delta) \leq\left(S_{2}, 0_{2}\right)$ then $\exists i, j \in \mathbb{N}^{*}:(Q, \delta)^{i} \subseteq\left(S_{2}, 0_{2}\right)^{j}$. It follows:

$$
\exists i, j \in \mathbb{N}^{*}:(Q, \delta)^{i} \subseteq\left(S_{2^{j}}, 0_{2^{j}}\right)
$$

$\Longrightarrow \quad \exists i \in I^{*}, \vec{s}=\left(s_{1}, \cdots, s_{i}\right) \in Q^{i}: \forall \vec{c}_{1}, \vec{c}_{2}, \vec{c}_{3} \in Q^{i} \quad \delta_{\mathrm{g}}^{i}\left(\vec{c}_{1}, \vec{c}_{2}, \vec{c}_{3}\right)=\vec{s}$

$\Longrightarrow \exists i \in \mathbb{N}^{*}, s_{0} \in Q: \forall \vec{c} \in Q^{2 i+1} \quad \delta^{i}(\vec{c})=s_{0}$

$\Longrightarrow(Q, \delta) \in N I L \vee|Q|=1$

Proposition 5 Let $(Q, \delta)$ be a CA. It holds:

$(Q, \delta) \sim\left(S_{2}, 0_{2}\right) \Longleftrightarrow(Q, \delta) \in N I L$

Proof If $(Q, \delta) \in N I L$ then, by definition, $\exists n \in N^{*}$ and $s_{0} \in Q$ such that $\forall \vec{c} \in Q^{2 n+1}$ $\delta^{n}(\vec{c})=s_{0}$. It follows that $(Q, \delta)^{n} \cong\left(S_{|Q|^{n}}, 0_{|Q|^{n}}\right)$ with $|Q|^{n}>1$ and therefore, by lemma 4 , $(Q, \delta) \sim\left(S_{2}, 0_{2}\right)$. The other implication corresponds to lemma 5 and to the fact that if $\left(S_{2}, 0_{2}\right) \leq(Q, \delta)$ then $|Q|>1$.

Proposition $6(Q, \delta)<\left(S_{2}, 0_{2}\right)$ if and only if $(Q, \delta)$ is isomorph to the minimum.

Proof Let us suppose that $(Q, \delta)<\left(S_{2}, 0_{2}\right)$ and $|Q|>1$. By lemma $5,(Q, \delta) \in N I L$, and therefore $(Q, \delta) \sim\left(S_{2}, 0_{2}\right) . \Rightarrow \Leftarrow$

Corollary 1 Given a CA $(Q, \delta)$, it is undecidable to know if $(Q, \delta) \leq\left(S_{2}, 0_{2}\right)$.

Proof By the fact that the nilpotency problem is undecidable ([3]). 


\subsection{Periodicity and Shift-Like Behavior}

Some other global properties concerning cyclic behavior are considered. First we say that a CA belongs to the class PER, and we call it periodic, if every configuration belongs to a cycle. More precisely,

$$
P E R=\left\{(Q, \delta):(|Q|>1) \wedge\left(\forall \mathcal{C} \in Q^{\mathbb{Z}}, \exists n \in N^{*}: G_{\delta}^{n}(\mathcal{C})=\mathcal{C}\right)\right\}
$$

On the other hand we introduce the $R_{S H I F T}$ and $L_{S H I F T}$ classes. In this case, for every configuration there exists an $n \in \mathbb{N}^{*}$ for which the configuration reappears $n$ cells shifted after $n$ time steps. In other words,

$$
\begin{aligned}
& R_{\text {SHIFT }}=\left\{(Q, \delta):(|Q|>1) \wedge\left(\forall \mathcal{C} \in Q^{\mathbb{Z}}, \exists n \in N^{*}:\left(\left(G_{\delta}\right)^{n}(\mathcal{C})\right)_{i}=\mathcal{C}_{i-n}\right)\right\} \\
& L_{\text {SHIFT }}=\left\{(Q, \delta):(|Q|>1) \wedge\left(\forall \mathcal{C} \in Q^{\mathbb{Z}}, \exists n \in N^{*}:\left(\left(G_{\delta}\right)^{n}(\mathcal{C})\right)_{i}=\mathcal{C}_{i+n}\right)\right\}
\end{aligned}
$$

As in the nilpotency case, for these classes the length of the cycles does not depend on the considered configurations. This result is stated in next lemma:

Lemma 6 It holds the following:

$$
\begin{aligned}
\text { PER } & =\left\{(Q, \delta):(|Q|>1) \wedge\left(\exists n \in \mathbb{N}^{*}, \forall \mathcal{C} \in Q^{\mathbb{Z}}: G_{\delta}^{n}(\mathcal{C})=\mathcal{C}\right)\right\} \\
R_{S H I F T} & =\left\{(Q, \delta):(|Q|>1) \wedge\left(\exists n \in \mathbb{N}^{*}, \forall \mathcal{C} \in Q^{\mathbb{Z}}:\left(\left(G_{\delta}\right)^{n}(\mathcal{C})\right)_{i}=\mathcal{C}_{i-n}\right)\right\} \\
L_{S H I F T} & =\left\{(Q, \delta):(|Q|>1) \wedge\left(\exists n \in \mathbb{N}^{*}, \forall \mathcal{C} \in Q^{\mathbb{Z}}:\left(\left(G_{\delta}\right)^{n}(\mathcal{C})\right)_{i}=\mathcal{C}_{i+n}\right)\right\}
\end{aligned}
$$

Proof Let $(Q, \delta) \in P E R$. Let us consider any configuration $\mathcal{C}^{*}$ in which all the words over $Q$ appear (it suffices to construct it as a suitable concatenation). Denoting the period of the cycle to which $\mathcal{C}^{*}$ belongs as $n^{*}$, it follows that

$$
\forall \vec{c}=\left(c_{-n^{*}}, \cdots, c_{0}, \cdots, c_{n^{*}}\right) \in Q^{2 n^{*}+1}: \delta^{n^{*}}(\vec{c})=c_{0}
$$

and therefore any other configuration $\mathcal{C} \in Q^{\mathbb{Z}}$ is $n^{*}$-periodic. For the $R_{S H I F T}$ and the $L_{S H I F T}$ classes the proof is exactly the same.

We introduce now the simplest periodic and shift-like CA: those having unitary length cycles. More precisely, let $n>1$ and let $\left(S_{n}, I_{n}\right),\left(S_{n}, \sigma_{n}\right)\left(S_{n}, \sigma_{n}^{-1}\right)$ be the CA such that, for all $x, y, z \in S_{n}$ :

$$
I_{n}(x, y, z)=y \quad \sigma_{n}(x, y, z)=x \quad \sigma_{n}^{-1}(x, y, z)=z
$$

It follows the same as for the nilpotency case. In fact, the proofs of next two propositions are completely equivalent to those of proposition 5 and proposition 6 :

Proposition 7 Let $(Q, \delta)$ be a CA. It holds:

$$
\begin{aligned}
(Q, \delta) \sim\left(S_{2}, I_{2}\right) & \Longleftrightarrow(Q, \delta) \in P E R \\
(Q, \delta) \sim\left(S_{2}, \sigma_{2}\right) & \Longleftrightarrow(Q, \delta) \in R_{S H I F T} \\
(Q, \delta) \sim\left(S_{2}, \sigma_{2}^{-1}\right) & \Longleftrightarrow(Q, \delta) \in L_{S H I F T}
\end{aligned}
$$

Proposition 8 Let $\left(S_{2}, \rho_{2}\right) \in\left\{\left(S_{2}, I_{2}\right),\left(S_{2}, \sigma_{2}\right),\left(S_{2}, \sigma_{2}^{-1}\right)\right\}$. Then $(Q, \delta)<\left(S_{2}, \rho_{2}\right)$ if and only if $(Q, \delta)$ is isomorph to the minimum.

Corollary 2 The canonical order on $(\mathrm{CA} / \sim)$ compatible with $\leq$ is partial. Moreover, the classes $R_{S H I F T}, L_{S H I F T}, N I L$, and PER are two by two incomparables.

Proof It suffices to consider non periodic configurations (for instance: $\cdots 0001000 \cdots$ ) and to notice that its behavior (its evolution) could never be simultaneously of two types. 


\section{Two Incomparable Infinite Chains with a Common Upper Bound}

A non trivial property concerning $(\mathrm{CA}, \leq)$ is proved in this section: the existence of two incomparable infinite chains with a common upper bound. The bound will correspond to a suitable composition of the CA that solves a slightly modified version of the well-known firing squad problem ([5]) with another one that simply transmits signals. The two chains are introduced in next definition:

Definition 2 Let $\left\{\left(S_{n}, \eta_{n}\right)\right\}_{n>1}$ and $\left\{\left(S_{n}, \mu_{n}\right)\right\}_{n>1}$ be two families of CA such that, for each $n>1$ :

$$
\begin{aligned}
& \eta_{n}(x, y, z)= \begin{cases}x & \text { if } x=y=z \\
0 & \text { otherwise }\end{cases} \\
& \mu_{n}(x, y, z)=\min \{x, y, z\}
\end{aligned}
$$

Before proving that previous families are incomparable and infinite chains, notice that there exists a pair of points belonging to different chains which are comparable. In fact, the initial points $\left(S_{2}, \eta_{2}\right)$ and $\left(S_{2}, \mu_{2}\right)$ are isomorph. On the other hand, they are located above the $N I L$ class as it is showed in next proposition:

Proposition 9 For all $\left(Q_{N I L}, \delta_{N I L}\right) \in N I L$ it holds:

$$
\begin{aligned}
& \left(Q_{N I L}, \delta_{N I L}\right)<\left(S_{2}, \eta_{2}\right) \\
& \left(Q_{N I L}, \delta_{N I L}\right)<\left(S_{2}, \mu_{2}\right)
\end{aligned}
$$

Proof Let $\left(Q_{N I L}, \delta_{N I L}\right) \in N I L$. First $\left(S_{2}, \eta_{2}\right) \not \leq\left(Q_{N I L}, \delta_{N I L}\right)$ because $\left(S_{2}, \eta_{2}\right)$ is not nilpotent. On the other hand, notice that $\left(S_{2}, 0_{2}\right) \subseteq\left(S_{2}, \eta_{2}\right)^{2}$ because it suffices to consider $\varphi: S_{2} \rightarrow\left(S_{2}\right)^{2}$ such that $\varphi(x)=(0 x)$.

Lemma 7 Let $n>1$. For all $i \in N^{*}$ it holds:

$$
\begin{aligned}
& \left|\left\{\vec{x} \in\left(S_{n}\right)^{i}:\left(\eta_{n}\right)_{\mathrm{g}}^{i}(\vec{x}, \vec{x}, \vec{x})=\vec{x}\right\}\right|=n \\
& \left|\left\{\vec{x} \in\left(S_{n}\right)^{i}:\left(\mu_{n}\right)_{\mathrm{g}}^{i}(\vec{x}, \vec{x}, \vec{x})=\vec{x}\right\}\right|=n
\end{aligned}
$$

Proof First, for all $x \in S_{n}$ :

$$
\begin{aligned}
& \left(\eta_{n}\right)_{\mathrm{g}}^{i}(x \cdots x, x \cdots x, x \cdots x)=x \cdots x \\
& \left(\mu_{n}\right)_{\mathrm{g}}^{i}(x \cdots x, x \cdots x, x \cdots x)=x \cdots x
\end{aligned}
$$

Let $\vec{x}=\left(x_{1} \cdots x_{i}\right) \in\left(S_{n}\right)^{i}$ be such that $\exists 1 \leq k<i$ such that $x_{k} \neq x_{k+1}$. Without loss of generality, let us assume $x_{k}>x_{k+1}$. It follows:

$$
\begin{aligned}
\left(\left(\eta_{n}\right)_{\mathrm{g}}^{i}(\vec{x}, \vec{x}, \vec{x})\right)_{k} & =0 \quad \neq x_{k} \\
\left(\left(\mu_{n}\right)_{\mathrm{g}}^{i}(\vec{x}, \vec{x}, \vec{x})\right)_{k} & \leq x_{k+1}<x_{k}
\end{aligned}
$$


Proposition 10 For all $n>1$ it holds:

$$
\begin{aligned}
& \left(S_{n}, \eta_{n}\right)<\left(S_{n+1}, \eta_{n+1}\right) \\
& \left(S_{n}, \mu_{n}\right)<\left(S_{n+1}, \mu_{n+1}\right)
\end{aligned}
$$

Proof First $\left(S_{n}, \eta_{n}\right) \leq\left(S_{n+1}, \eta_{n+1}\right)$ because $\left.\eta_{n+1}\right|_{S_{n}}=\eta_{n}$. Let us suppose that there exist $i, j \in \mathbb{N}^{*}$ such that $\left(S_{n+1}, \eta_{n+1}\right)^{i} \subseteq\left(S_{n}, \eta_{n}\right)^{j}$. Let $\varphi:\left(S_{n+1}\right)^{i} \rightarrow\left(S_{n}\right)^{j}$ be the suitable injection. It follows that, if $\vec{x} \in\left(S_{n+1}\right)^{i}$ is such that $\left(\eta_{n+1}\right)_{\mathrm{g}}^{i}(\vec{x}, \vec{x}, \vec{x})=\vec{x}$ then $\left(\eta_{n}\right)_{\mathrm{g}}^{j}(\varphi(\vec{x}), \varphi(\vec{x}), \varphi(\vec{x}))=\varphi(\vec{x})$ and we contradict lemma 7 . For $\left(S_{n}, \mu_{n}\right)<\left(S_{n+1}, \mu_{n+1}\right)$ the argument is exactly the same.

Lemma 8 Let $i, n \in N^{*}$ with $n>1$ and let $\vec{a}=\left(a_{1} \cdots a_{i}\right) \in\left(S_{n}\right)^{i}$. It holds:

$$
\begin{aligned}
& \left(\eta_{n}\right)_{\mathrm{g}}^{i}(\vec{a}, \vec{a}, \vec{a})= \begin{cases}\left(a_{1} \cdots a_{1}\right) & \text { if } a_{1}=a_{2}=\cdots=a_{i} \\
(0 \cdots 0) & \text { otherwise }\end{cases} \\
& \left(\mu_{n}\right)_{\mathrm{g}}^{i}(\vec{a}, \vec{a}, \vec{a})=\left(a^{*} \cdots a^{*}\right), \text { where } a^{*}=\min \left\{a_{1}, \cdots, a_{i}\right\}
\end{aligned}
$$

Proof It suffices to notice that, for all $\vec{x}=\left(x_{-i} \cdots x_{0} \cdots x_{i}\right) \in\left(S_{n}\right)^{2 i+1}$ it holds:

$$
\begin{aligned}
& \left(\eta_{n}\right)^{i}\left(x_{-i} \cdots x_{0} \cdots x_{i}\right) \neq 0 \Longleftrightarrow x_{-i}=\cdots=x_{0}=\cdots=x_{i} \neq 0 \\
& \left(\mu_{n}\right)^{i}\left(x_{-i} \cdots x_{0} \cdots x_{i}\right)=\min \left\{x_{-i}, \cdots, x_{0}, \cdots, x_{i}\right\}
\end{aligned}
$$

Proposition 11 For all $n>2, m>2$ it holds: $\left(S_{m}, \mu_{m}\right) \not \leq\left(S_{n}, \eta_{n}\right)$.

Proof Let us suppose that there exist $i, j \in N^{*}$ such that $\left(S_{m}, \mu_{m}\right)^{i} \subseteq\left(S_{n}, \eta_{n}\right)^{j}$ and let us denote $\varphi:\left(S_{m}\right)^{i} \rightarrow\left(S_{n}\right)^{j}$ the suitable injection. It follows that:

$$
\forall x \in S_{m}, \exists \varphi_{x} \in S_{n} \text { such that } \varphi(x \cdots x)=\left(\varphi_{x} \cdots \varphi_{x}\right)
$$

In fact,

$$
\begin{aligned}
\varphi(x \cdots x) & =\varphi\left(\left(\mu_{m}\right)_{\mathrm{g}}^{i}(x \cdots x, x \cdots x, x \cdots x)\right) \\
& =\left(\eta_{n}\right)_{\mathrm{g}}^{j}(\varphi(x \cdots x), \varphi(x \cdots x), \varphi(x \cdots x)) \\
& =\left(\varphi_{x} \cdots \varphi_{x}\right)(\text { by lemma } 8)
\end{aligned}
$$

Let $x \in S_{m}$ be such that $0 \leq x<m-1$ and $\varphi(x \cdots x) \neq(0 \cdots 0)$. It follows:

$$
\begin{aligned}
\varphi(x \cdots x) & =\varphi\left(\left(\mu_{m}\right)_{\mathrm{g}}^{i}(m-1 \cdots m-1, x \cdots x, m-1 \cdots m-1)\right) \\
& =\left(\eta_{n}\right)_{\mathrm{g}}^{j}\left(\varphi_{m-1} \cdots \varphi_{m-1}, \varphi_{x} \cdots \varphi_{x}, \varphi_{m-1} \cdots \varphi_{m-1}\right) \\
& =(0 \cdots 0) \Rightarrow \Leftarrow \square
\end{aligned}
$$

Proposition 12 For all $n>2, m>2$ it holds: $\left(S_{n}, \eta_{n}\right) \not \leq\left(S_{m}, \mu_{m}\right)$.

Proof Let us suppose that there exist $i, j \in \mathbb{N}^{*}$ such that $\left(S_{n}, \eta_{n}\right)^{i} \subseteq\left(S_{m}, \mu_{m}\right)^{j}$ and let us denote $\varphi:\left(S_{n}\right)^{i} \rightarrow\left(S_{m}\right)^{j}$ the suitable injection. As in the proof of proposition 11, it holds the following:

$$
\forall x \in S_{n}, \exists \varphi_{x} \in S_{m} \text { such that } \varphi(x \cdots x)=\left(\varphi_{x} \cdots \varphi_{x}\right)
$$


Let $x, y \in S_{n}$ be such that $\varphi_{x}>\varphi_{y}>0$ and $y \neq 0$. It follows:

$$
\begin{aligned}
\varphi(0 \cdots 0) & =\varphi\left(\left(\eta_{n}\right)_{\mathrm{g}}^{i}(x \cdots x, y \cdots y, x \cdots x)\right) \\
& =\left(\mu_{m}\right)_{\mathrm{g}}^{j}\left(\varphi_{x} \cdots \varphi_{x}, \varphi_{y} \cdots \varphi_{y}, \varphi_{x} \cdots \varphi_{x}\right) \\
& =\left(\varphi_{y} \cdots \varphi_{y}\right) \\
& =\varphi(y \cdots y) \Rightarrow \Leftarrow
\end{aligned}
$$

Now in order to obtain an upper bound for the two previously introduced infinite chains we are going to compose a pair of CA. One of them is related to the classical firing squad problem introduced in [5] and which consists to design a CA capable to synchronize "as soon as possible" an array of cells of arbitrary size. In lemma 9 a result that appears in [6] concerning a slightly modified version of the original problem known as two-ends firing squad is formally stated:

Lemma $9[6]$ There exists a $\mathrm{CA}\left(Q_{F S}, \delta_{F S}\right)$ such that $\left\{G_{l}, G_{r}, q_{0}\right\} \subseteq Q_{F S}$ and which satisfies for all $n \in N^{*}$ the following:

- $\left(\delta_{F S}\right)_{\mathrm{g}}^{n+2}\left(G_{l} q_{0} \cdots q_{0} G_{r}, G_{l} q_{0} \cdots q_{0} G_{r}, G_{l} q_{0} \cdots q_{0} G_{r}\right)=\left(G_{l} q_{0} \cdots q_{0} G_{r}\right)$

- For all substring $\vec{x}$ of the triple concatenation $\left(G_{l} q_{0} \cdots q_{0} G_{r} G_{l} q_{0} \cdots q_{0} G_{r} G_{l} q_{0} \cdots q_{0} G_{r}\right) \in$ $\left(Q_{F S}\right)^{3(n+2)}$ such that $|\vec{x}|=2 k+1$ with $k<(n+2)$, it holds:

$$
\left(\delta_{F S}\right)^{k}(\vec{x}) \neq q_{0}
$$

In next figure it is represented the case $n+2=4$ :

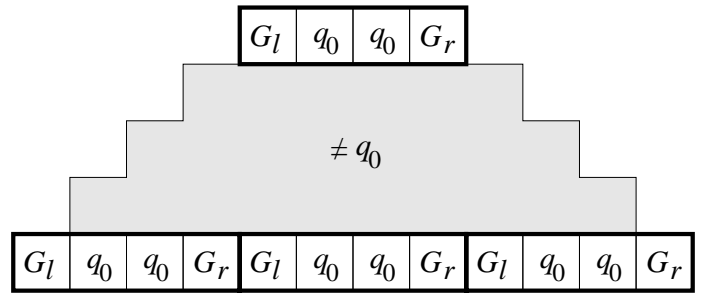

Fig. 3 Two-ends firing squad.

The CA to be composed with $\left(Q_{F S}, \delta_{F S}\right)$ is introduced in next definition. Its cells simply transmit the signals (or states) coming from its left (resp. right) neighbor to its right (resp. left) neighbor keeping only its own information. More precisely,

Definition 3 Let $Q$ be an arbitrary set of states. We define the CA $\left(S_{Q}^{\text {signal }}, \delta_{Q}^{\text {signal }}\right)$ such that $S_{Q}^{\text {signal }}=Q^{3}$ and for all $x, y, z \in S_{Q}^{\text {signal }}$ :

$$
\delta_{Q}^{\text {signal }}(x, y, z)=\left(x_{l} y_{c} z_{r}\right)
$$

where all the states of $S_{Q}^{\text {signal }}$ are denoted $s=\left(s_{l} s_{c} s_{r}\right)$. 
The composition CA is almost the "superposition" of the two previously introduced CA (with the set of signals $Q=\{0,1\}$ ). The exception is done at the last step of the firing squad period: the only triplets of 1 signals not destroyed (transformed into 0 ) are those arriving simultaneously to a cell. Formally:

Definition 4 Let $(\mathcal{Q}, \mathcal{D})$ be the CA such that $\mathcal{Q}=Q_{F S} \times S_{\{0,1\}}^{\text {signal }}$ and, for all $\left(x_{1}, x_{2}\right),\left(y_{1}, y_{2}\right),\left(z_{1}, z_{2}\right)$ $\in \mathcal{Q}$ :

$$
\mathcal{D}\left(\left(x_{1}, x_{2}\right),\left(y_{1}, y_{2}\right),\left(z_{1}, z_{2}\right)\right)= \begin{cases}\left(\delta_{F S}\left(x_{1}, y_{1}, z_{1}\right), 000\right) & \text { if } \begin{array}{l}
\delta_{F S}\left(x_{1}, y_{1}, z_{1}\right)=q_{0} \\
\text { and } \delta_{\{0,1\}}^{\text {signal }}\left(x_{2}, y_{2}, z_{2}\right) \neq(1
\end{array} \\
\left(\delta_{F S}\left(x_{1}, y_{1}, z_{1}\right), \delta_{\{0,1\}}^{\text {signal }}\left(x_{2}, y_{2}, z_{2}\right)\right) & \text { otherwise }\end{cases}
$$

Proposition 13 For all $n>1:\left(S_{n}, \eta_{n}\right)<(\mathcal{Q}, \mathcal{D})$.

Proof Let $n \in \mathbb{N}^{*} .\left(S_{n}, \eta_{n}\right) \subseteq(\mathcal{Q}, \mathcal{D})^{n+1}$ by the injection $\varphi: S_{n} \rightarrow \mathcal{Q}^{n+1}$ that follows:

$$
\begin{aligned}
& \varphi(0)=\left(\left(G_{l}, 000\right),\left(q_{0}, 000\right), \cdots,\left(q_{0}, 000\right),\left(G_{r}, 000\right)\right) \\
& \varphi(x)=\left(G_{l}, 000\right), \underbrace{\left(q_{0}, 000\right), \cdots,\left(q_{0}, 000\right),\left(q_{0}, 111\right)},\left(q_{0}, 000\right), \cdots,\left(q_{0}, 000\right),\left(G_{r}, 000\right))
\end{aligned}
$$

In fact, as it is shown in figure 4 ,

$$
\mathcal{D}_{\mathrm{g}}^{n+1}(\varphi(x), \varphi(y), \varphi(z))= \begin{cases}\varphi(x) & \text { if } \varphi(x)=\varphi(y)=\varphi(z)=\varphi\left(\eta_{n}(x, y, z)\right) \\ \varphi(0) & \text { otherwise }\end{cases}
$$

On the other hand $(\mathcal{Q}, \mathcal{D}) \not\left(S_{n}, \eta_{n}\right)$ because $\left(S_{n+1}, \eta_{n+1}\right) \not \leq\left(S_{n}, \eta_{n}\right)$.
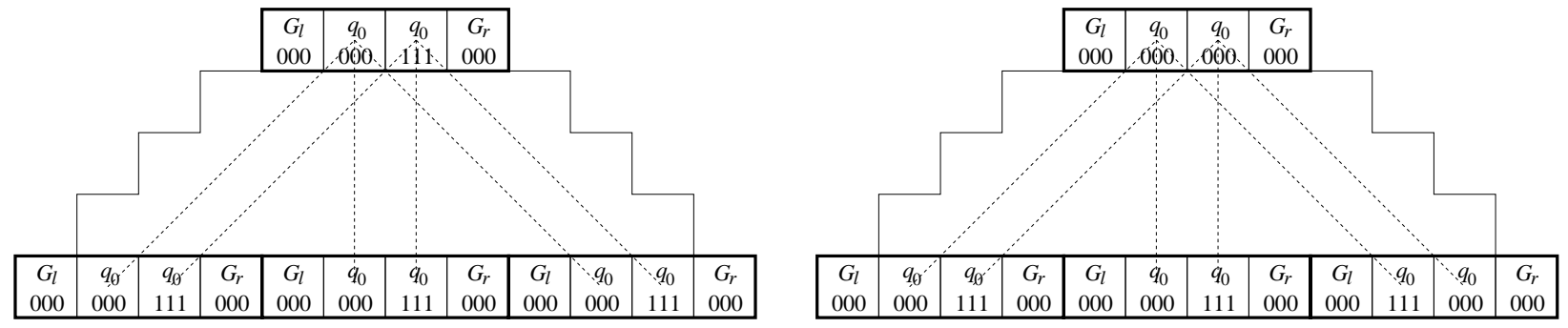

Fig. 4 "Simulating" by $(\mathcal{Q}, \mathcal{D})^{4}$ the transitions of $\left(S_{3}, \eta_{3}\right)$ for $(2,2,2)$ and $(2,2,1)$.

Proposition 14 For all $n>1:\left(S_{n}, \mu_{n}\right)<(\mathcal{Q}, \mathcal{D})$.

Proof Let $n \in \mathbb{N}^{*} .\left(S_{n}, \eta_{n}\right) \subseteq(\mathcal{Q}, \mathcal{D})^{n+1}$ by the injection $\varphi: S_{n} \rightarrow \mathcal{Q}^{n+1}$ that follows:

$$
\begin{aligned}
& \varphi(0)=\left(\left(G_{l}, 000\right),\left(q_{0}, 000\right), \cdots,\left(q_{0}, 000\right),\left(G_{r}, 000\right)\right) \\
& \varphi(x)=\left(G_{l}, 000\right), \underbrace{\left(q_{0}, 111\right), \cdots,\left(q_{0}, 111\right)}_{x \text { with } 0<x \leq n-1},\left(q_{0}, 000\right), \cdots,\left(q_{0}, 000\right),\left(G_{r}, 000\right))
\end{aligned}
$$

In fact, as for $\eta_{n}$ :

$$
\mathcal{D}_{\mathrm{g}}^{n+1}(\varphi(x), \varphi(y), \varphi(z))=\varphi(\min \{x, y, z\})=\varphi\left(\mu_{n}\{x, y, z\}\right)
$$

On the other hand $(\mathcal{Q}, \mathcal{D}) \not\left(S_{n}, \mu_{n}\right)$ because $\left(S_{n+1}, \mu_{n+1}\right) \not \leq\left(S_{n}, \mu_{n}\right)$. 


\section{An Unbounded Infinite Chain}

In this section we prove that $(\mathrm{CA}, \leq)$ has no maximum. In other words, for any $\mathrm{CA}$, the subsystems of all its grouped instances will never cover all the CA classes. This result allows us to conclude that, in a "grouping sense", there is no universal CA. The proof is implicitly based on the existence of an unbounded infinite chain obtained after "processing" the next one:

Definition $5\left\{\left(S_{n}, \Delta_{n}\right)\right\}_{n>1}$ is the family of CA such that, for each $n>1$ :

$$
\Delta_{n}(x, y, z)= \begin{cases}x & \text { if } x=z \\ y & \text { if } x \neq z\end{cases}
$$

Notice that the bottom of previous family is located above the NIL class or more precisely, as it is shown in next proposition, above the $\mathrm{CA}\left(S_{2}, \eta_{2}\right)$ introduced in definition 2 :

Proposition $15\left(S_{2}, 0_{2}\right)<\left(S_{2}, \eta_{2}\right)<\left(S_{2}, \Delta_{2}\right)$.

Proof First it suffices to notice, as it is shown in figure 5 , that $\left(S_{2}, \eta_{2}\right) \subseteq\left(S_{2}, \Delta_{2}\right)^{2}$ by the injection $\varphi: S_{2} \rightarrow\left(S_{2}\right)^{2}$ such that $\varphi(x)=(0 x)$. On the other hand, let us suppose that there exist $i, j \in \mathbb{N}^{*}$ such that $\left(S_{2}, \Delta_{2}\right)^{i} \subseteq\left(S_{2}, \eta_{2}\right)^{j}$. By lemma $2,\left(S_{2}, \Delta_{2}\right)^{2 i} \subseteq\left(S_{2}, \eta_{2}\right)^{2 j}$. By lemma 7 ,

$$
\left|\left\{\vec{x} \in\left(S_{2}\right)^{2 j}:\left(\eta_{2}\right)_{\mathrm{g}}^{2 j}(\vec{x}, \vec{x}, \vec{x})=\vec{x}\right\}\right|=2
$$

However,

$$
\left|\left\{\vec{x} \in\left(S_{2}\right)^{2 i}:\left(\Delta_{2}\right)_{\mathrm{g}}^{2 i}(\vec{x}, \vec{x}, \vec{x})=\vec{x}\right\}\right| \geq 4
$$

\begin{tabular}{|c|c|c|c|c|c|c|c|c|c|c|c|c|c|c|c|c|c|c|c|c|c|c|c|}
\hline & & 0 & 0 & & & & & 0 & 0 & & & & & 0 & 0 & & & & & \begin{tabular}{l|l}
0 \\
\end{tabular} & 0 & & \\
\hline & 0 & 0 & 0 & 0 & & & 0 & 0 & 0 & 0 & & & 0 & 0 & 0 & 0 & & & 0 & \begin{tabular}{l|l|}
0 \\
\end{tabular} & 0 & 0 & \\
\hline \begin{tabular}{|l|}
0 \\
\end{tabular} & \begin{tabular}{l|l}
0 \\
\end{tabular} & 0 & 0 & 0 & 0 & 0 & 1 & 0 & 0 & 0 & 0 & 0 & 0 & 0 & \begin{tabular}{l|l}
1 \\
\end{tabular} & 0 & 0 & 0 & 0 & \begin{tabular}{l|l|}
0 \\
\end{tabular} & 0 & 0 & 1 \\
\hline & & 0 & 0 & & & & & 0 & 0 & & & & & \begin{tabular}{l|l}
0 \\
\end{tabular} & 0 & & & & & 0 & 1 & & \\
\hline & 0 & 1 & 0 & 0 & & & 0 & 0 & 0 & 0 & & & 0 & \begin{tabular}{l|l|}
0 \\
\end{tabular} & 0 & 1 & & & 0 & \begin{tabular}{l|l}
1 \\
\end{tabular} & 0 & 1 & \\
\hline 0 & 1 & 0 & 1 & 0 & 0 & 0 & 1 & 0 & 0 & 0 & 1 & 0 & 0 & 0 & 1 & 0 & 1 & 0 & 1 & 0 & 1 & 0 & 1 \\
\hline
\end{tabular}

because for all $x, y \in S_{2}:\left(\Delta_{2}\right)_{\mathrm{g}}^{2 i}(x y \cdots x y, x y \cdots x y, x y \cdots x y)=(x y \cdots x y)$

Fig. 5 Embedding $\left(S_{2}, \eta_{2}\right)$ into $\left(S_{2}, \Delta_{2}\right)^{2}$.

The following is the key result of the present section. It says, in a general way, that the $\Delta_{n}$ 's transition functions are too complicated to be simulated by grouped instances of a CA. This impossibility is based on the fact that, for any grouped instance of a CA, any cell belonging to a block has not access during the interaction process to the whole information contained on the neighbor blocks. Formally: 
Proposition 16 For every $\mathrm{CA}(Q, \delta)$ and for every $n \in \mathbb{N}^{*}$ such that $n>|Q|$ it holds:

$$
\forall i \in N^{*}:\left(S_{n}, \Delta_{n}\right) \nsubseteq(Q, \delta)^{i}
$$

Proof Suppose that there exist $(Q, \delta)$ and $n, i \in \mathbb{N}^{*}$ such that $n>|Q|$ and $\left(S_{n}, \Delta_{n}\right) \subseteq$ $(Q, \delta)^{i}$. Then, by definition, there exists an injection $\varphi: S_{n} \rightarrow Q^{i}$ such that:

$$
\forall x, y, z \in S_{n}: \varphi\left(\Delta_{n}(x, y, z)\right)=\delta_{\mathrm{g}}^{i}(\varphi(x), \varphi(y), \varphi(z))
$$

Let $i_{0}$ be the smallest index of $Q^{i}$ for which there exist at least two elements of $\varphi\left(S_{n}\right)$ having different values (see figure 6 ). Formally:

$$
i_{0}=\min \left\{k \in \mathbb{N}^{*}: \exists\left(x_{1}, \cdots, x_{i}\right),\left(y_{1}, \cdots, y_{i}\right) \in \varphi\left(S_{n}\right) \text { such that } x_{k} \neq y_{k}\right\}
$$

Notice that $i_{0} \in\{1, \cdots i\}$ is well defined because $\left|S_{n}\right|>1$. It follows:

$$
\forall \vec{x}, \vec{z} \in \varphi\left(S_{n}\right): \vec{x} \neq \vec{z} \Rightarrow x_{i_{0}} \neq z_{i_{0}}
$$

In fact, suppose that there exist $\vec{x}, \vec{z} \in \varphi\left(S_{n}\right)$ with $\vec{x} \neq \vec{z}$ such that $x_{i_{0}}=z_{i_{0}}$. By construction of $i_{0}$ there always exists $\vec{y} \in \varphi\left(S_{n}\right)$ such that $x_{i_{0}} \neq y_{i_{0}}$. On the other hand:

$$
\begin{aligned}
x_{i_{0}}=\left(\delta_{\mathrm{g}}^{i}(\vec{x}, \vec{y}, \vec{x})\right)_{i_{0}} & =\delta^{2 i+1}\left(x_{i_{0}}, \cdots, x_{i}, y_{1} \cdots, y_{i}, x_{1}, \cdots, x_{i_{0}}\right) \\
& =\delta^{2 i+1}\left(x_{i_{0}}, \cdots, x_{i}, y_{1} \cdots, y_{i}, z_{1}, \cdots, z_{i_{0}}\right) \\
& =\left(\delta_{\mathrm{g}}^{i}(\vec{x}, \vec{y}, \vec{z})\right)_{i_{0}}=y_{i_{0}} \Rightarrow \Leftarrow
\end{aligned}
$$

Finally it follows that $\beta: S_{n} \rightarrow Q$ with $\beta(x)=(\varphi(x))_{i_{0}}$ is an injection and therefore $n \leq|Q| . \Rightarrow \Leftarrow$

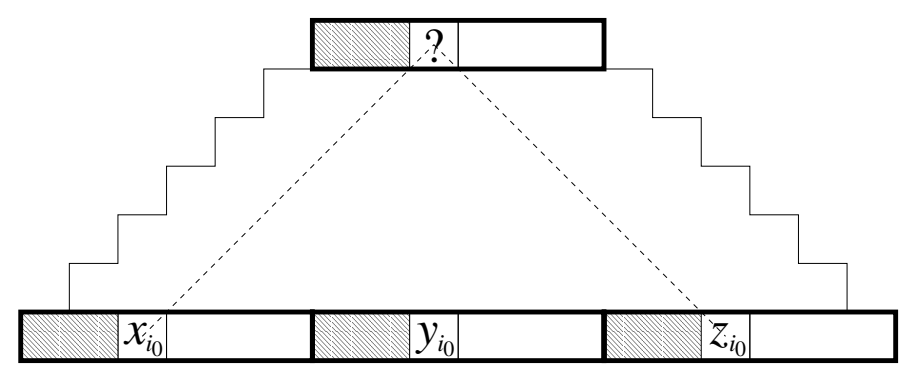

Fig. 6 Cell $i_{0}$ and the information it may access.

In order to conclude that $(\mathrm{CA}, \leq)$ admits no maximum we are going to show that every $\mathrm{CA}$ is contained in all the grouped instances of a suitable composition of itself with the CA that transmits signals introduced in definition 3. More precisely,

Lemma 10 For any $\mathrm{CA}(Q, \delta)$ there exists a normalized version $(Q, \delta)^{*}=\left(Q^{*}, \delta^{*}\right)$ satisfying, for all $i \in \mathbb{N}^{*}:(Q, \delta) \subseteq\left(Q^{*}, \delta^{*}\right)^{i}$. 
Proof Let us denote $B$ as a state not belonging to any CA. Let $(Q, \delta)$ be a CA. We define $Q^{*}=S_{\{Q \cup\{B\}\}}^{\text {signal }}$ and for all $x, y, z \in Q^{*}$ :

$$
\delta^{*}(x, y, z)= \begin{cases}\left(\delta\left(x_{l}, y_{c}, z_{r}\right), \delta\left(x_{l}, y_{c}, z_{r}\right), \delta\left(x_{l}, y_{c}, z_{r}\right)\right) & \text { if } x_{l}, y_{c}, z_{r} \in Q \\ \delta_{\{Q \cup\{B\}\}}^{\text {signal }}(x, y, z) & \text { otherwise }\end{cases}
$$

For any $i \in N^{*}$, the injection $\varphi: Q \rightarrow\left(Q^{*}\right)^{i}$ that allows us to conclude the lemma is the following: $\varphi(x)=((x x x),(B B B), \cdots(B B B))$ (see figure 7$)$.

\begin{tabular}{|c|c|c|c|c|c|c|c|c|}
\hline & & \begin{tabular}{|l}
$\delta(x y z)$ \\
$\delta(x y z)$ \\
$\delta(x y z)$ \\
\end{tabular} & $B B B$ & $B B B$ & \multirow[b]{2}{*}{$B z B$} & & \\
\hline & & $x B B$ & ByB & $B B z$ & $y B B$ & & & \\
\hline & $x B B$ & BBy & $B y B$ & $y B B$ & $B B z$ & $B z B$ & $z B B$ & \\
\hline$x x x$ & $B B B$ & $B B B$ & yyy & $B B B$ & $B B B$ & $z z z$ & $B B B$ & $B B B$ \\
\hline
\end{tabular}

Fig. 7 Embedding $(Q, \delta)$ into $\left(Q^{*}, \delta^{*}\right)^{i}$ for $i=3$.

Proposition 17 For every $\mathrm{CA}(Q, \delta)$ and for every $n>|Q|:\left(S_{n}^{*}, \Delta_{n}^{*}\right) \not \subset(Q, \delta)$.

Proof Suppose that there exist $(Q, \delta), n>|Q|$ and $i, j \in \mathbb{N}^{*}$ such that $\left(S_{n}^{*}, \Delta_{n}^{*}\right)^{i} \subseteq(Q, \delta)^{j}$. Then, by lemma $10,\left(S_{n}, \Delta_{n}\right) \subseteq(Q, \delta)^{j}$, which contradicts proposition 16 .

Corollary $3(\mathrm{CA}, \leq)$ has no maximum.

Proposition 18 It holds, for all $n \geq 1:\left(S_{n}^{*}, \Delta_{n}^{*}\right)<\left(S_{(n+1)^{3}+1}^{*}, \Delta_{(n+1)^{3}+1}^{*}\right)$.

Proof $\left(S_{n}^{*}, \Delta_{n}^{*}\right) \leq\left(S_{(n+1)^{3}+1}^{*}, \Delta_{(n+1)^{3}+1}^{*}\right)$ because $S_{n}^{*} \subseteq S_{(n+1)^{3}+1}^{*}$ and $\left.\Delta_{(n+1)^{3}+1}^{*}\right|_{n} ^{*}=\Delta_{n}^{*}$. On the other hand, let us suppose that $\left(S_{(n+1)^{3}+1}^{*}, \Delta_{(n+1)^{3}+1}^{*}\right) \leq\left(S_{n}^{*}, \Delta_{n}^{*}\right)$. Considering that $\left|S_{n}^{*}\right|=(n+1)^{3}$ we contradict proposition 17 .

Proposition 19 For every $\mathrm{CA}(Q, \delta)$ there exists another one $(\tilde{Q}, \tilde{\delta})$ such that $(Q, \delta)<$ $(\tilde{Q}, \tilde{\delta})$.

Proof It suffices to consider $(\tilde{Q}, \tilde{\delta})=(Q, \delta) \otimes\left(S_{|Q|+1}^{*}, \Delta_{|Q|+1}^{*}\right)$. By proposition $3(Q, \delta) \leq$ $(\tilde{Q}, \tilde{\delta})$. On the other hand, if $(\tilde{Q}, \tilde{\delta}) \leq(Q, \delta)$ then $\left(S_{|Q|+1}^{*}, \Delta_{|Q|+1}^{*}\right) \leq(Q, \delta) . \Rightarrow \Leftarrow$ 


\section{Concluding Remarks}

In this paper we have introduced a preorder on the set of CA. We have shown that on the bottom of this structure very natural equivalence classes are located. These classes remind us the first two well-known Wolfram ones because they capture global (or dynamical) properties as nilpotency or periodicity. Non trivial properties as the undecidability of $\leq$ and the existence of bounded infinite chains were also proved. Finally, it was shown that $(\mathrm{CA}, \leq)$ admits no maximum. This result allowed us to conclude that, in a "grouping sense", there is no universal CA. Further research should be guided by the following ideas:

- A deeper understanding of the $(\mathrm{CA}, \leq)$ structure could help us to develop a complexity notion on $\mathrm{CA}$.

- If a CA $A$ is a grouped instance of $B$, then $B$ could be thought as an efficient codification of $A$. It seems very natural to look for characterizations of reducible $\mathrm{CA}$ and to study the associated computational complexity problem.

- The concept of intrinsic or self-referenced simulation on CA was introduced in [1]. It simply means that we "simulate directly a CA without passing through Turing machines". In [4] appears an intrinsic universal CA working on quasi linear time but restricted to totalistic transitions. Our relation $\leq$ may be interpreted as a particular kind of simulation working on real time. A clarifying study about the different notions of simulations (and the time they take) should be done in the future.

\section{References}

[1] Albert J., Culik II K. A simple universal cellular automaton and its one-way and totalisting version, Complex Systems, 1 (1987), 1-16.

[2] Culik II K., Pachl J., Yu S. On the limit sets of cellular automata, SIAM J. Computing, 18 (1989), 831-842.

[3] Kari J. The nilpotency problem of one-dimensional cellular automata, SIAM J. Computing, 21 (1992), 571-586.

[4] Martin B. A universal cellular automaton in quasi-linear time and its $s-m-n$ form, Theoretical Computer Science, 123(2) (1994), 199-237.

[5] Moore E.F. Sequential machines, selected papers, Addison Wesley Reading Mass., (1964), 213-214.

[6] Mazoyer J., Reimen N. A linear speed-up theorem for cellular automata, Theoretical Computer Science, 101 (1992), 59-98.

[7] Smith III A.R. Simple computation-universal cellular spaces, Journal ACM, 18 (1971), 339-353. 
[8] Wolfram S. Universality and complexity in cellular automata, Physica D 10 (1984), $1-35$. 